\title{
The Six-Item Cognitive Impairment Test Is Associated with Adverse Outcomes in Acutely Hospitalized Older Patients: A Prospective Cohort Study
}

\author{
Jacinta A. Lucke ${ }^{a}$ b Roos C. van der Mast ${ }^{c, d}$ Jelle de Gelder ${ }^{a}$ Noor Heim ${ }^{a}$ \\ Bas de Groot ${ }^{b}$ Simon P. Mooijaart ${ }^{a}$ e Gerard J. Blauw ${ }^{a}$ \\ a Section of Geriatrics, Department of Internal Medicine, Leiden University Medical Center, \\ Leiden, The Netherlands; ${ }^{b}$ Department of Emergency Medicine, Leiden University Medical \\ Center, Leiden, The Netherlands; ' ${ }^{2}$ eppartment of Psychiatry, Leiden University Medical Center, \\ Leiden, The Netherlands; ${ }^{d}$ Collaborative Antwerp Psychiatric Research Institute (CAPRI), \\ University of Antwerp, Antwerp, Belgium; ${ }^{\mathrm{e}}$ Institute for Evidence-Based Medicine in Old Age \\ (IEMO), Leiden, The Netherlands
}

\section{Keywords}

Cognitive impairment $\cdot$ Hospital admission $\cdot$ Functional impairment $\cdot$ Acute care $\cdot$ Six-Item Cognitive Impairment Test · Mortality · Hospital length of stay · Older patients · Geriatrics

\begin{abstract}
Aim: The study aim was to investigate whether cognitive impairment, measured by the SixItem Cognitive Impairment Test (6-CIT), is an independent predictor of adverse outcomes in acutely hospitalized older patients. Methods: This was a prospective multicenter study including acutely hospitalized patients aged 70 years and older. Multivariable logistic regression was used to investigate whether impaired cognition (6-CIT $\geq 11$ points) was an independent predictor of 90-day adverse outcome, a composite measure of functional decline and mortality. Secondary endpoints were hospital length of stay, new institutionalization, and in-hospital mortality. Results: In total, 196 (15.6\%) of 1,252 included patients had a 6-CIT $\geq 11$. Median age was 80 years (interquartile range $74-85$ ). Patients with impaired cognition had higher rates of 90 -day adverse outcome (41.7\% compared to $30.3 \%$ in 1,056 not cognitively impaired patients, $p=0.009$ ). Impaired cognition was a predictor of 90 -day adverse outcome with a crude odds ratio (OR) of 1.64 (95\% Cl 1.13-2.39), but statistical significance was lost when fully corrected for possible confounders (OR 1.44, 95\% Cl 0.98-2.11). For all secondary outcomes, impaired cognition was an independent predictor. Conclusions: In the acute hospital setting, the 6-CIT is associated with 90-day adverse outcome and is an independent predictor of hospital length of stay, new institutionalization, and in-hospital mortality.
\end{abstract}

(c) 2018 The Author(s)

Published by S. Karger AG, Basel 


\section{Introduction}

Acute hospitalized older patients have a high risk of adverse outcomes [1], and cognitively impaired older patients are at an even greater risk compared to patients with normal cognition [2]. Cognitive impairment can be caused by dementia, delirium, hypoperfusion of the brain, or by a combination of these disorders. Impaired cognition is highly prevalent in acutely hospitalized older patients, but is frequently missed by doctors and nurses. Whichever the cause, professional caretakers should be vigilant for the presence of cognitive impairment as it calls for measures to prevent adverse events and to ensure safety when patients are hospitalized.

To date, in most studies investigating predictors of outcome among hospitalized older patients, the Mini-Mental State Examination (MMSE) [3] was used to assess cognitive impairment, often in combination with premorbid ADL dependency [4-7]. However, a cognition test to be used in the acute hospital setting should be short, easy to administer and feasible when patients are unable to write. While the MMSE is considered the gold standard test, it has limitations due to the relatively lengthy time it takes to administer, its interaction with the level of education and the requirement to be able to write. In comparison with the MMSE, the Six-Item Cognitive Impairment Test (6-CIT) [8] takes only 2-3 min [9], is not influenced by educational level, can be used in bed-bound patients who are unable to write, and showed comparable test characteristics. If adverse outcome of acutely hospitalized older patients could be predicted by impaired cognition as assessed with the 6-CIT, it would be a suitable test to improve identification of older patients at risk for adverse events in the acute setting and to help identify their needs.

Therefore, the aim of the present study was to investigate the association of impaired cognition, as measured with the 6-CIT, and adverse outcomes in acutely hospitalized older patients. A cohort study among three hospitals in the Netherlands was conducted, and a distinction was made between short-term adverse outcomes (in-hospital mortality, new institutionalization, and prolonged length of hospital stay) and long-term adverse outcomes (90-day functional decline and mortality).

\section{Materials and Methods}

\section{Study Design and Setting}

This was a secondary analysis using the data of a prospective multicenter study the "Recovery Care Programme" (HerstelZorgProgramma) [10]. A detailed description of the study design can be found in the article by Heim et al. [10]. In summary, this was an observational cohort study in which data were prospectively collected during 3 consecutive years, in the same season. Three secondary care facilities (Alrijne Hospital, Leiden; Alrijne Hospital, Leiderdorp; and Bronovo Hospital, The Hague) and one tertiary care hospital (Leiden University Medical Center, Leiden) participated.

The medical ethics committee of the Leiden University Medical Center (LUMC) waived the need for ethical approval as data were collected to improve patient care. All patients provided written informed consent, and data were treated anonymously.

\section{Participants}

Patients aged 70 years or older who were admitted to one of the four study hospitals were assessed for inclusion. Two secondary care hospitals and one tertiary care hospital included both acutely admitted and planned patients (wards of orthopedics, neurology, urology, and surgery). One secondary care hospital included only acutely admitted patients. 
For the analyses, the wards of orthopedics, urology, and surgery were combined into "surgery," and the departments of internal medicine, neurology, and cardiology were combined into "medical."

Patients were excluded if they stayed in the hospital for less than $48 \mathrm{~h}$ and if they were not able to perform the study interview within $72 \mathrm{~h}$ after admission. Patients who had an MMSE of $<19$, indicating severe cognitive impairment, and had no caregiver present during the interview were also excluded because they could not provide informed consent. For the present secondary analysis, only acutely admitted patients, from both medical and surgical departments, were included.

\section{Data Collection}

Patients were interviewed on the wards by a trained nurse with a series of questionnaires. After 90 days, patients were sent follow-up questionnaires by mail, to be self-administered. Patients who did not respond were contacted by telephone.

\section{Cognitive Function}

The 6-CIT[8] contains items on orientation, attention, and memory with a range from 0 to 28; a score $\geq 11$ indicates cognitive impairment. The 6 -CIT showed a good correlation with the MMSE, and a cut-off point of 11 corresponded to an MMSE of $\leq 23$ [9]. The 6-CIT score was used to classify older people into those with (score $\geq 11$ ) and without (score $<11$ ) cognitive impairment, a cut-off that has been recommended in the literature.

MMSE evaluates overall cognitive functions, such as orientation, memory, attention, calculation [3], ranging from 0 to 30 , and a score $\leq 23$ indicating cognitive impairment. Scores $<19$ indicate severe cognitive impairment.

\section{Functional Status}

The Katz Index on Independence in Activities of Daily Living [11] (Katz-ADL) was administered to quantify functional status. The Katz-ADL contains 6 yes/no items on whether a patient is independent in bathing, dressing, transferring from bed to chair, eating, going to the toilet, and the use of incontinence products. A score of $\geq 2$ points means dependency in ADL [12].

\section{Demographics}

Data on age, sex, and self-reported living situation were registered by the research nurse. Also, the medical specialism and hospital where the patient was treated were registered.

\section{Outcomes}

Primary Outcome

The primary outcome was defined as a composite endpoint of adverse outcome, containing self-reported functional decline (by increasing one point in Katz-ADL) after 90 days and/or mortality within 90 days. Mortality was verified in the hospital files, by the healthcare insurer or were reported by family members. The cut-off point of $\geq 1$ point increase in Katz-ADL was chosen because this results in a clinically relevant decrease in independency [12].

\section{Secondary Outcomes}

Three secondary outcomes were investigated: in-hospital mortality, new institutionalization directly after hospital admission, and prolonged hospital length of stay (LOS). New institutionalization was defined as moving from an independent living situation to assisted home care facilities directly after discharge from the hospital. Prolonged hospital LOS was defined as an LOS of 7 days or longer. 


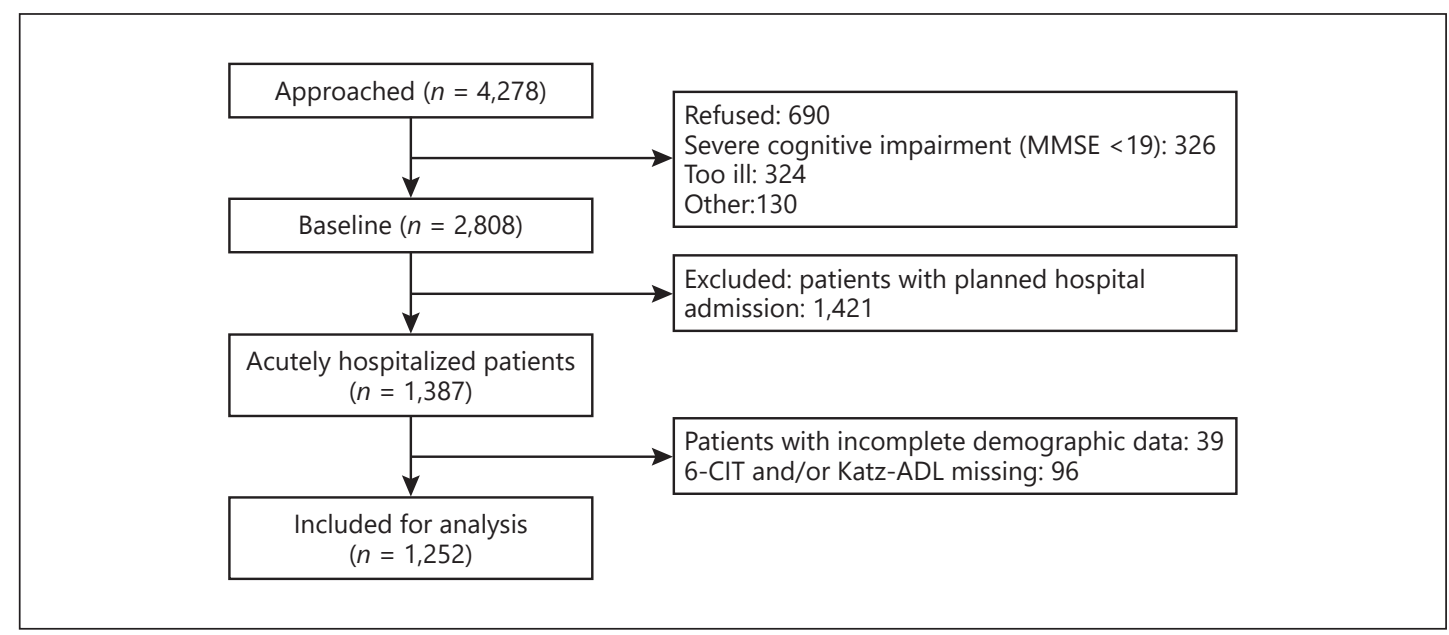

Fig. 1. Flowchart of study population. MMSE, Mini-Mental State Examination; 6-CIT, Six-Item Cognitive Impairment Test; Katz-ADL, Katz index of activities of daily living.

\section{Data Analysis}

Data are displayed as percentages, means, and standard deviations for normally distributed variables or as medians with interquartile ranges for non-normally distributed variables. Independent $t$ tests and $\chi^{2}$ tests were used to assess equality of groups when variables were normally distributed and with Mann-Whitney U tests for non-normally distributed variables. The association between 6-CIT and primary and secondary outcomes was calculated using crosstabs and $\chi^{2}$ tests. Patients were divided into two groups for analysis, using the 6 -CIT $(\leq 10, \geq 11)$ at baseline. Univariable logistic regression was used to assess the crude association between 6-CIT and primary and secondary outcomes.

Two multivariable logistic regression models were used to assess whether 6-CIT was an independent predictor of adverse outcome. The first model was corrected for age and sex. In the second model, the association of interest was also corrected for living situation and specialism, to correct for baseline functional status and type of disease. The general rule of thumb that there should be a minimum of 10 events per possible variable in the model was used.

Statistical significance was defined by $95 \%$ confidence intervals (CI) excluding 1.0 or $p<$ 0.05. All statistical analyses were performed using IBM SPSS Statistics package (version 23; IBM, New York, NY, USA).

\section{Results}

\section{Baseline Characteristics}

A total of 1,252 patients was included in this study (Fig. 1), of which the baseline characteristics are shown in Table 1 . The majority of patients was female $(n=710,56.8 \%)$, and median age was 80 years (interquartile range 74-85). In 196 patients (15.6\%), the 6-CIT score was $\geq 11$, indicating cognitive impairment. In Table 1 , it is shown that patients with cognitive impairment were older, less frequently male, and more often lived in an assisted living facility, compared to patients with a lower 6-CIT score. 
Table 1. Baseline characteristics of the total study population and stratified according to 6-CIT score

\begin{tabular}{|c|c|c|c|c|}
\hline Characteristic & $\begin{array}{l}\text { All patients } \\
n=1,252\end{array}$ & $\begin{array}{l}6-\mathrm{CIT} \leq 10 \\
n=1,056\end{array}$ & $\begin{array}{l}6-\mathrm{CIT} \geq 11 \\
n=196\end{array}$ & $p$ value \\
\hline Age, years & $80(74-85)$ & $79(74-84)$ & $82(78-87)$ & $<0.001$ \\
\hline Male & $542(43.2)$ & $476(45.1)$ & $66(33.7)$ & 0.003 \\
\hline Living situation ${ }^{\mathrm{a}}$ & & & & $<0.001$ \\
\hline Independent, with others & $591(47.5)$ & $519(49.4)$ & $72(36.9)$ & \\
\hline Independent, alone & $522(41.9)$ & $443(42.2)$ & $79(40.5)$ & \\
\hline Assisted living facility & $132(10.6)$ & $88(8.4)$ & $44(22.6)$ & \\
\hline Specialism $^{\mathrm{b}}$ & & & & 0.740 \\
\hline Surgical & $770(61.7)$ & $647(61.5)$ & $123(62.8)$ & \\
\hline Medical & $478(38.3)$ & $405(38.5)$ & $73(37.2)$ & \\
\hline Hospital & & & & $<0.001$ \\
\hline LUMC & $205(16.4)$ & $166(15.7)$ & 39 (19.9) & \\
\hline Alrijne - Leiden & $297(23.7)$ & $240(22.7)$ & $57(29.1)$ & \\
\hline Alrijne - Leiderdorp & $375(30.0)$ & $308(29.2)$ & $67(34.2)$ & \\
\hline Bronovo & $375(30.0)$ & $342(32.4)$ & $33(16.8)$ & \\
\hline Katz-ADL ${ }^{\mathrm{c}}$ & $1(0-3)$ & $0(0-2)$ & $1(0-4)$ & $<0.001$ \\
\hline 6-CIT & $4(0-8)$ & $2(0-5)$ & $14(12-18)$ & n.a \\
\hline $\mathrm{MMSE}^{\mathrm{d}}$ & $27(24.3-29)$ & $28(26-29)$ & $21(19-24)$ & n.a. \\
\hline
\end{tabular}

Data are presented as $n(\%)$ or median (interquartile range). 6-CIT, Six-Item Cognitive Impairment Test; Katz-ADL, Katz index of activities of daily living; n.a., not applicable. ${ }^{a}$ Number of values $1,245 .{ }^{b}$ Number of values 1,248. ${ }^{\mathrm{c}}$ Number of values $1,252 .{ }^{\mathrm{d}}$ Number of values 892.

Table 2. Crude outcomes for total study population and according to 6-CIT score

\begin{tabular}{|c|c|c|c|c|}
\hline & $\begin{array}{l}\text { Total } \\
n=1,252\end{array}$ & $\begin{array}{l}6 \text {-CIT } \leq 10 \\
n=1,056\end{array}$ & $\begin{array}{l}6 \text {-CIT } \geq 11 \\
n=196\end{array}$ & $p$ value \\
\hline \multicolumn{5}{|l|}{ Primary outcome } \\
\hline 90-day adverse outcome ${ }^{a}$ & 311 (31.8) & $256(30.3)$ & $55(41.7)$ & 0.009 \\
\hline \multicolumn{5}{|l|}{ Secondary outcomes } \\
\hline LOS $\geq 7$ days $^{b}$ & $563(45.1)$ & $455(43.3)$ & $108(55.4)$ & 0.002 \\
\hline New institutionalization ${ }^{c}$ & $67(7.4)$ & $46(5.8)$ & $21(18.8)$ & $<0.001$ \\
\hline In-hospital mortality ${ }^{\mathrm{d}}$ & $20(1.6)$ & $12(1.2)$ & $8(4.1)$ & 0.003 \\
\hline
\end{tabular}

Data are presented as $n(\%) .6$-CIT, Six-Item Cognitive Impairment Test; LOS, length of stay. ${ }^{\text {a Number of }}$ values $977 .{ }^{\mathrm{b}}$ Number of values $1,247 .{ }^{\mathrm{c}}$ Number of values $905 .{ }^{\mathrm{d}}$ Number of values 1,236.

\section{Primary and Secondary Outcomes}

A total of 311 (31.8\%) patients suffered from 90-day mortality or functional decline. Table 2 shows the incidence of various negative outcomes over strata of 6-CIT. More than $30 \%$ of patients with a 6-CIT $\leq 10$ suffered from 90 -day mortality or functional decline, in comparison to $41.7 \%$ patients with a 6 -CIT $\geq 11(p=0.009)$. Patients with impaired cognition had a prolonged hospital stay of $\geq 7$ days more frequently ( $n=455,43.3 \%$ vs. $n=108,55.4 \%$, respectively; $p=0.002$ ) and were more often institutionalized after hospital admission, compared to those with a normal cognition. Also, in-hospital mortality was higher in cognitively impaired patients compared to cognitively normal patients $(n=12,1.2 \%$ vs. $n=8,4.1 \%$, respectively; $p=0.003$ ). 
Table 3. Association between the 6-CIT and adverse outcomes in older acutely hospitalized patients

\begin{tabular}{|c|c|c|c|}
\hline & $6-\mathrm{CIT} \leq 10$ & $\begin{array}{l}6-\mathrm{CIT} \geq 11 \\
\text { OR }(95 \% \mathrm{CI})\end{array}$ & $p$ value \\
\hline \multicolumn{4}{|l|}{ Primary outcome: 90-day functional decline and mortality ${ }^{a}$} \\
\hline Crude & 1 (Ref) & $1.64(1.13-2.39)$ & 0.010 \\
\hline Model 1 - corrected for age and sex & 1 (Ref) & $1.48(1.01-2.17)$ & 0.045 \\
\hline Model 2 - age, sex, living situation, and specialism & 1 (Ref) & $1.44(0.98-2.11)$ & 0.066 \\
\hline \multicolumn{4}{|l|}{ Secondary outcome: $\geq 7$ days LOS ${ }^{b}$} \\
\hline Crude & 1 (Ref) & $1.63(1.20-2.22)$ & 0.002 \\
\hline Model 1 - corrected for age and sex & 1 (Ref) & $1.51(1.11-2.07)$ & 0.009 \\
\hline Model 2 - age, sex, living situation, and specialism & 1 (Ref) & $1.54(1.12-2.12)$ & 0.008 \\
\hline \multicolumn{4}{|l|}{ Secondary outcome: new institutionalization ${ }^{c}$} \\
\hline Crude & 1 (Ref) & $3.74(2.14-6.56)$ & $<0.001$ \\
\hline Model 1 - corrected for age and sex & 1 (Ref) & $2.94(1.64-5.28)$ & $<0.001$ \\
\hline Model 2 - age, sex, living situation, and specialism & 1 (Ref) & $3.45(1.89-6.31)$ & $<0.001$ \\
\hline \multicolumn{4}{|l|}{ Secondary outcome: in-hospital mortality ${ }^{\mathrm{d}}$} \\
\hline Crude & 1 (Ref) & $3.67(1.48-9.10)$ & 0.005 \\
\hline Model 1 - corrected for age and sex & 1 (Ref) & $3.18(1.26-8.05)$ & 0.015 \\
\hline Model 2 - age, sex, living situation, and specialism & 1 (Ref) & $3.11(1.21-7.99)$ & 0.018 \\
\hline
\end{tabular}

OR, odds ratio; 95\% CI, 95\% confidence interval; 6-CIT, Six-Item Cognitive Impairment Test. ${ }^{\text {a }}$ Patients included for analysis: 977. ${ }^{\mathrm{b}}$ Patients included for analysis: 1,247. ${ }^{\mathrm{c}}$ Patients included for analysis: 905. d Patients included for analysis: 1,236.

\section{Independent Predictors}

Patients with impaired cognition as assessed with the 6-CIT had a 1.6 times increased risk of mortality or functional decline after 90 days (odds ratio 1.64, 95\% CI 1.13-2.39). When corrected for age and sex, this association was still observed, but after correction for living situation and treating medical specialism, statistical significance was lost (Table 3). Patients with impaired cognition were also at increased risk of prolonged hospital stay and of a 3-fold increased risk of being institutionalized, independent of age, sex, living situation, and medical specialism. Finally, impaired cognition was independently associated with in-hospital mortality.

\section{Discussion}

The present study shows that, in acutely hospitalized older patients with impaired cognition, as defined by a 6 -CIT score $\geq 11$, there is an association with increased risk of 90-day adverse outcome (functional decline and mortality). We interpret the fact that statistical significance was lost after adjustment as a result of adding more variables in the model, as the estimate remained virtually unchanged. Further, it is shown that impaired cognition is independently associated with a hospital LOS $\geq 7$ days as well as increased in-hospital mortality and institutionalization.

Our findings are in line with the literature, reporting an association between impaired cognition and functional decline, mortality, and hospital LOS [2, 6, 13-15]. Care providers often experience barriers in administering a cognition test in the acute setting. If such a test would be used on a regular basis, nurses and doctors could take instant tailor-made actions, e.g. history taking, explaining treatment, involving relatives at an early stage, and taking 
measures to prevent or treat delirium, which might prevent adverse outcomes in older patients. Several screening tools for measuring cognitive dysfunction have been proposed $[14,16]$. The 6-CIT appears to be an instrument that can be easy and quickly applied, has a low chance of interpretation error, and can also be administered in patients who are unable to read, write, or perform lengthy tests $[10,16]$. In this study, we further showed that the 6-CIT is an independent predictor of adverse outcomes, such as prolonged hospitalization, institutionalization, and in-hospital mortality. Because of this combination of test characteristics and association with adverse outcomes, it might be a good tool to implement in daily practice.

In our study, we used the 6-CIT for screening of cognitive impairment, irrespective of its cause, and showed that patients who are cognitively impaired have an increased risk of adverse outcomes. Dementia and delirium are the main causes of cognitive impairment in older patients, but they can be difficult to diagnose and differentiate in the acute setting. As recently proposed by Jackson et al. [17], cognitive impairment per se in acute hospital admissions is common and associated with poor health outcomes. Also, in a recent paper by Reynish et al. [18], it was shown that there are no big differences in adverse outcomes between patients with different types of cognitive impairment. Therefore, when managing acutely ill older patients, it is important to treat them based on their needs, rather than on a specific diagnosis. Medical staff needs to be vigilant and assess cognition on a routine basis. A short test such as the 6-CIT could facilitate this. In case of impaired cognition, the patient should be treated optimally in terms of optimizing the care process, providing environmental adjustments, and minimizing harm [17]. The proactive diagnosis of impaired cognition per se, whatever the specific underlying diagnosis, is likely to improve patient experience and outcomes, because the caregiver can focus on interventions, rather than on diagnostics. Furthermore, cognitive impairment should be considered when developing health care policies for improvement of outcomes such as hospital length of stay, new institutionalization, and in-hospital mortality.

We did not find an independent association of cognitive impairment with long-term outcome, probably because after adding more variables to the model, borderline significance was lost. However, the estimates remained virtually unchanged.

The present study has several limitations. First, the exclusion of patients with an MMSE of $<19$ points leads to an underestimation of the prevalence of cognitive impairment. However, in patients with subtler cognitive impairment, the 6-CIT adds possibly unknown clinical information, while severely cognitive impaired patients are recognized relatively easily (e.g., nursing home patients with known dementia). Secondly, the $22 \%$ loss to follow-up after 90 days may have led to selection bias. However, the patients who were lost to follow-up were likely more cognitively impaired and frail, which leads to an underestimation of the association found in this study.

Major strengths of this study are the large sample size and multicenter design. Also, the duration of the study, in 3 consecutive years, during similar months renders the study more robust as temporary environmental effects are less likely to have influenced the data. The combination of both long- and short-term outcomes is another strength of this study.

In conclusion, cognitive impairment measured with the 6-CIT is associated with 90-day adverse outcomes in acutely admitted older patients and is an independent predictor of prolonged hospital length of stay, institutionalization, and in-hospital mortality. This emphasizes the importance of routinely screening for cognitive impairment in this vulnerable patient group. Further research should focus on integrating cognition in risk-screening tools and investigate whether interventions for patients with impaired cognition improve clinically relevant outcomes. 
Lucke et al.: The 6-CIT and Adverse Outcomes in Acutely Hospitalized Older Patients

\section{Statement of Ethics}

All procedures performed in this study involving human participants were in accordance with the ethical standards of the institutional research committee and with the 1964 Helsinki declaration and its later amendments or comparable ethical standards. The Medical Ethics Committee of the Leiden University Medical Center waived the need for ethical approval as the study was conducted to improve patient care. All patients provided written informed consent and data were treated anonymously.

\section{Disclosure Statement}

The authors declare that they have no conflicts of interest.

\section{Funding Sources}

This work was supported by the Netherlands Organization for Health Research and Development (ZonMw project number 313060201). The sponsor had no role in the design of the study, the collection, analysis, and interpretation of the data, and did not influence the writing of the manuscript.

\section{Author Contributions}

G.J.B. and R.C.M. were involved in the design of the study, obtaining funding, and the execution of the study. N.H. collected the data and created the databases. J.A.L. performed the statistical analysis and drafted the paper. J.G. and B.G. assisted with the statistical analyses. B.G., G.J.B., R.C.M., and S.P.M. advised during the writing process. All authors contributed to its revision and gave approval of the final version of the article.

\section{References}

1 Aminzadeh F, Dalziel WB: Older adults in the emergency department: a systematic review of patterns of use, adverse outcomes, and effectiveness of interventions. Ann Emerg Med 2002;39:238-247.

2 Provencher V, Sirois MJ, Ouellet MC, Camden S, Neveu X, Allain-Boule N, Emond M; Canadian Emergency Team Initiative on Mobility in Aging: Decline in activities of daily living after a visit to a Canadian emergency department for minor injuries in independent older adults: are frail older adults with cognitive impairment at greater risk? J Am Geriatr Soc 2015;63:860-868.

3 Folstein MF, Folstein SE, McHugh PR: "Mini-mental state." A practical method for grading the cognitive state of patients for the clinician. J Psychiatr Res 1975;12:189-198.

4 De Buyser SL, Petrovic M, Taes YE, Vetrano DL, Onder G: A multicomponent approach to identify predictors of hospital outcomes in older in-patients: a multicentre, observational study. PLoS One 2014;9:e115413.

5 Vetrano DL, Landi F, De Buyser SL, Carfi A, Zuccala G, Petrovic M, Volpato S, Cherubini A, Corsonello A, Bernabei $\mathrm{R}$, et al: Predictors of length of hospital stay among older adults admitted to acute care wards: a multicentre observational study. Eur J Intern Med 2014;25:56-62.

6 Avila-Funes JA, Amieva H, Barberger-Gateau P, Le Goff M, Raoux N, Ritchie K, Carriere I, Tavernier B, Tzourio C, Gutierrez-Robledo LM, et al: Cognitive impairment improves the predictive validity of the phenotype of frailty for adverse health outcomes: the three-city study. J Am Geriatr Soc 2009;57:453-461.

7 Buurman BM, Hoogerduijn JG, de Haan RJ, Abu-Hanna A, Lagaay AM, Verhaar HJ, Schuurmans MJ, Levi M, de Rooij SE: Geriatric conditions in acutely hospitalized older patients: prevalence and one-year survival and functional decline. PLoS One 2011;6:e26951.

8 Katzman R, Brown T, Fuld P, Peck A, Schechter R, Schimmel H: Validation of a short Orientation-MemoryConcentration Test of cognitive impairment. Am J Psychiatry 1983;140:734-739. 
9 Tuijl JP, Scholte EM, de Craen AJ, van der Mast RC: Screening for cognitive impairment in older general hospital patients: comparison of the Six-Item Cognitive Impairment Test with the Mini-Mental State Examination. Int J Geriatr Psychiatry 2012;27:755-762.

10 Heim N, van Fenema EM, Weverling-Rijnsburger AW, Tuijl JP, Jue P, Oleksik AM, Verschuur MJ, Haverkamp JS, Blauw GJ, van der Mast RC, et al: Optimal screening for increased risk for adverse outcomes in hospitalised older adults. Age Ageing 2015;44:239-244.

11 Katz S, Ford AB, Moskowitz RW, Jackson BA, Jaffe MW: Studies of illness in the aged. The index of ADL: a standardized measure of biological and psychosocial function. JAMA 1963;185:914-919.

12 De Rooij SE, et al: Praktijkgids “Kwetsbare Ouderen." The Hague, VMSzorg, 2009.

13 Hustey FM, Meldon SW, Smith MD, Lex CK: The effect of mental status screening on the care of elderly emergency department patients. Ann Emerg Med 2003;41:678-684.

14 Carpenter CR, Bassett ER, Fischer GM, Shirshekan J, Galvin JE, Morris JC: Four sensitive screening tools to detect cognitive dysfunction in geriatric emergency department patients: brief Alzheimer's Screen, Short Blessed Test, Ottawa 3DY, and the caregiver-completed AD8. Acad Emerg Med 2011;18:374-384.

15 Han JH, Eden S, Shintani A, Morandi A, Schnelle J, Dittus RS, Storrow AB, Ely EW: Delirium in older emergency department patients is an independent predictor of hospital length of stay. Acad Emerg Med 2011;18:451457.

16 Yokomizo JE, Simon SS, Bottino CM: Cognitive screening for dementia in primary care: a systematic review. Int Psychogeriatr 2014;26:1783-1804.

17 Jackson TA, Gladman JR, Harwood RH, MacLullich AM, Sampson EL, Sheehan B, Davis DH: Challenges and opportunities in understanding dementia and delirium in the acute hospital. PLoS Med 2017;14:e1002247.

18 Reynish EL, Hapca SM, De Souza N, Cvoro V, Donnan PT, Guthrie B: Epidemiology and outcomes of people with dementia, delirium, and unspecified cognitive impairment in the general hospital: prospective cohort study of 10,014 admissions. BMC Med 2017;15:140. 